\title{
3 Research Square

\section{A Rare Case of IgG4-related Disease Manifesting as an Ulcerated Gastric Cancer Complicated With Gastric Mucormycosis}

\section{Yuting Zhan}

Second Xiangya Hospital Department of Pathology

\section{Yang Yang}

Second Xiangya Hospital Department of Pathology

Hongmei Zheng

Second Xiangya Hospital Department of Pathology

\section{Yuting Zhang}

Second Xiangya Hospital Department of Pathology

\section{Li Gu}

Second Xiangya Hospital , Department of Digestive Medicine

\section{Dongliang Chen}

The Affiliated Zhuzhou Hospital, Xiangya Medical College of Central South University

\section{Songqing Fan ( $\nabla$ songqingfan@csu.edu.cn )}

Second Xiangya Hospital

\section{Case Report}

Keywords: IgG4-related disease, gastric ulcer, mucormycosis

Posted Date: September 7th, 2021

DOl: https://doi.org/10.21203/rs.3.rs-818871/v1

License: (c) (i) This work is licensed under a Creative Commons Attribution 4.0 International License. Read Full License 


\section{Abstract}

\section{Background}

IgG4-related disease (IgG4-RD) is a novel clinical entity that mimics many malignant, infectious, and inflammatory disorders. Many organs or tissues are now known to be involved in IgG4-RD; however, there has been no report about lgG4-RD of stomach with fungal infection.

\section{Case presentation}

We reported a 56-year-old male with IgG4-RD manifesting as ulcerated gastric cancer complicated with gastric mucormycosis. Both clinical and imaging manifestations were atypical, in clinical, it was highly suspected that the disease is a malignant tumor as ulcerated gastric cancer. However, no pathological evidence of malignancy could be found in repeated biopsies and surgically excised gastric specimen. Histological examination showed ulcer and inflammatory granulation with lymphocytes and plasma cells in sheets, inflammation of vascular wall, formation of new fibrous tissue and inflammatory necrosis with hyphae and spores. The plasma cells had immunohistochemically strong staining for CD38, CD138, kappa, lambda, IgG and IgG4. And notably, IgG4/IgG positive plasma cell ratio was more than $40 \%$, kappa/lambda positive staining ratio was approximately $1: 1$. Gastric mucormycosis was diagnosed with hyphae and spores confirmed by Gomori's methenamine silver staining and immunofluorescence and confocal laser-scanning microscopy. IgG4-RD manifesting as ulcerated gastric cancer complicated with gastric mucormycosis was diagnosed by multidisciplinary team discussion from gastroenterology, radiology and pathology department. The patient vomited blood three times for the following month. An emergent exploratory laparotomy was conducted, and a mass with $10 \times 9 \times 8 \mathrm{~cm}$ adhered closely to adjacent organs could be touched in lesser curvature of gastric antrum. Anastomotic leakage occurred after the surgery, symptomatic treatments (negative pressure drainage and antibiotics) were applied without steroids and anti-fungus drugs. The patient gradually recovered and was advised to consult gastroenterology department for further steroids and anti-fungus drugs treatment.

Conclusions

To our knowledge, it is the first report about IgG4-related disease manifesting a gastric ulcer with mucormycosis infection.

\section{Highlights}

First report about the diagnosed as an IgG4-related disease manifesting as ulcerated gastric cancers complicated with gastric mucormycosis

Systematically reviewed pathological features in biopsy and surgical specimen of IgG4-related disease Multidisciplinary team discussion is necessary and indispensable for the diagnosis 


\section{Background}

IgG4-related disease (IgG4-RD) is a chronic multi-organ-involved fibroinflammatory condition characterized by tendency to form tumefactive lesions, increased serum levels of IgG4 (often but not always) and tissue infiltration by dense lymphocytes and IgG4-positive plasma cells. IgG4-RD is always confused with neoplasm, infectious and inflammatory disorders clinically and radiologically ${ }^{[1,2]}$. Many organs and tissues are now known to be involved in IgG4-RD including pancreas, biliary duct, lacrimal/salivary glands, lymph node, lung, liver, kidney, thyroid gland, gastrointestinal tract, prostate, hypophysis, stomach, skin, aorta, joint, retroperitoneum, meninges and pleura ${ }^{[2-6]}$. To our knowledge, there has been no report about IgG4-RD of stomach with fungal infection. Here we report a case of IgG4$\mathrm{RD}$ manifesting as an ulcerated gastric cancer complicated with gastric mucormycosis.

\section{Presentation Of Cases}

A 56-year-old male presented with fever and sought for medical advice in local hospital in August 2018. Laboratory tests revealed anemia (hemoglobin $81 \mathrm{~g} / \mathrm{L}$, reference range $130-175 \mathrm{~g} / \mathrm{L}$ ) and computer tomography (CT) scan indicated gastric antral region irregular thickening, dilatation of bile ducts of liver left lobe, and nodule in left upper lung. Positron emission tomography-computed tomography (PET-CT) showed radioactive accumulations in the above position as well as retroperitoneal lymph nodes (Fig. 1CF). A huge ulcer with $50 \times 45 \mathrm{~mm}$ was observed by esophagogastroduodenoscopy (EGD), whereas no evidences of pathological diagnosis of stomach carcinoma. The patient was treated with proton pump inhibitors (PPI) and antibiotics to resist Helicobacter pylori for two weeks, nevertheless, ulcer still existed $(50 \times 50 \mathrm{~mm})$ and no evidence of malignant cells pathologically as well. A laparoscopic removal of the mass was conducted with rapid pathological diagnosis for three times, neither the mass nor lymph node was positive for malignant tumor cells. No tumor cells were found by immunohistochemistry (IHC) detecting in the postoperative tissues again. Repeated fever occurred after operation with intermittent anorexia, meteorism, vomiting, fatigue, diaphoresis and melena until February 2019.

During this period, the patient was in hospital for twice, laboratory tests still revealed anemia (hemoglobin $69 \mathrm{~g} / \mathrm{L}$ and $74 \mathrm{~g} / \mathrm{L}$ respectively in January and February 2019), CT indicated the alteration of body and the antrum of stomach (February 2019), and mass biopsy observed fungi but no tumor cells once again (February 2019). Treated with PPI, antibiotics, octreotide, nutrition agents and drugs for relieving symptoms, the patient remained to complain uncomfortable. The patient undergone appendectomy at the age of 24 and he was diagnosed to type 2 diabetes in 2017. Pulmonary shadow was found in 2015 by routine physical examination. He had history of tobacco smoking and alcohol drinking for 27 years, and quit smoking for 3 years. There were no history of allergies, genetically related diseases, and infectious diseases.

The patient visited gastroenterology department of our hospital (The Second Xiangya Hospital, Central South University) in March 2019 for clear diagnosis and treatment. Left subcostal subxiphoid mass could be palpated by physical examination. Main positive laboratory tests were as follows: hemoglobin $81 \mathrm{~g} / \mathrm{L}$, 
globulin $49.4 \mathrm{~g} / \mathrm{L}$ (reference range $20-40 \mathrm{~g} / \mathrm{L}$ ), erythrocyte sedimentation rate $(\mathrm{ESR}) 121 \mathrm{~mm} / \mathrm{h}$ (reference range $1-15 \mathrm{~mm} / \mathrm{h}$ ), IgG4 22.4g/L (reference range 0.030-2.010g/L), lgG 22.6g/L (reference range 7.51$15.6 \mathrm{~g} / \mathrm{L}$ ), lgM 0.3g/L (reference range $0.46-3.04 \mathrm{~g} / \mathrm{L}$ ), lgE $>6000 \mathrm{ng} / \mathrm{mL}$ (reference range $0-691 \mathrm{ng} / \mathrm{ml}$ ), Helicobacter pylori weakly positive (blood test), faecal occult blood tests weakly positive. The following laboratory results were negative: blood amylase, lipase, G test, GM test, extractable nuclear antigen (ENA), antinuclear antibody (ANA), anti-neutrophil cytoplasmic antibodies (ANCA), anti-protease 3 (PR3), antimyeloperoxidase (MPO), glomerular basement membrane antibody (GBM), HIV, HBV, and EBV. Multiple lymph nodes of bilateral axillas (largest $5 \times 4 \mathrm{~mm}$ ) and inguinal regions (largest $9 \times 4 \mathrm{~mm}$ ) were found by ultrasound. Contrast-enhanced CT indicated that the appearance of gastric wall thickening, status of perigastric and retroperitoneal lymph node enlargement, and nodule in left upper lung with double lungs exudation (Fig. 1A and 1B). Sputum smear found Gram-positive cocci and bacillus as well as Gramnegative cocci, but no fungus, whereas esophageal mucosa smear could see hyphae and spores.

\section{Pathological Findings}

EGD was conducted again with mass biopsy (Fig. 2A-D). Microscopically, ulcer (Fig. 3A) and inflammatory granulation (Fig. 3B) could be seen with lymphocytes and plasma cells in sheets (Fig. 3C), inflammation of vascular wall (Fig. 3D), formation of new fibrous tissue (Fig. 3E) and inflammatory necrosis with hyphae and spores also existed (Fig. 3F). Immunohistochemically, there were cells with positive staining for CD38 (Fig. 4A), CD138 (Fig. 4B), CD10, MUM1, kappa (Fig. 4C), lambda (Fig. 4D) IgG (Fig. 4E) and IgG4 (Fig. 4F); focal positive staining for CD5, CD3, Bcl-2, and TiA-1 were observed; there were negative staining for Bcl-6, P53, GrB, PAX-5, CD30, CD20, CD56. Ki-67 index was 40\%. The positive expression of $\mathrm{CK}$ in normal gastric epithelial cells, but no tumor cell was found again. Worthy to mention was that IgG4/IgG was more than $40 \%$. Mucormycosis was diagnosed with hyphae and spores confirmed by Gomori's methenamine silver staining (Fig. 5A and Fig. 5B) and immunofluorescence and confocal laser-scanning microscopy (Fig. 5C). Due to the suspicions of lymphoma in clinical and image, we further completed gene rearrangement of $\mathrm{B}$ lymphocytes, and the result was negative. Therefore, it was highly suspected for this patient of IgG4-RD presenting as an atypical gastric ulcer complicated with gastric mucormycosis through the multidisciplinary team discussion.

The patient vomited blood three times for the following month (dark-red, approximately $150 \mathrm{~mL}, 10 \mathrm{~mL}$ and $70 \mathrm{~mL}$ respectively). An emergent exploratory laparotomy was conducted, and a mass with $10 \times 9 \times 8 \mathrm{~cm}$ adhered closely to adjacent organs could be touched in lesser curvature of gastric antrum (Supplementary Fig. 1A and 1B). The gastrectomy, abdominal lymph node biopsy and jejunal nutrition tube placement were then conducted.

Pathological examination showed the inflammatory granulation with lymphocytes and plasma cells in sheets (Supplementary Fig. 1C), inflammation of vascular wall (Supplementary Fig. 1D), formation of new fibrous tissue (Supplementary Fig. 1E) and multinuclear giant cells (Supplementary Fig. 1F). The plasma cells had immunohistochemically strong staining for CD38 (Supplementary Fig. 1G), CD138 (Supplementary Fig. 1H), kappa (Supplementary Fig. 1I), lambda (Supplementary Fig. 1J), IgG 
(Supplementary Fig. 1K) and IgG4 (Supplementary Fig. 1L). And the IgG4/ IgG ratio was more than 40\%; kappa/lambda ratio was approximately $1: 1 ; \mathrm{Ki}-67$ index was 70\%. Tuberculosis was negative by polymerase chain reaction (PCR). Anastomotic leakage occurred after the surgery, treated with negative pressure drainage and antibiotics (Meropenem, following Levofloxacin) for a month. During this period, no steroids and anti- fungus drugs were used. The latest laboratory tests revealed lgG4 22.4g/L one month after the surgery. The patient was advised to consult gastroenterology department for further steroids and anti-fungus drugs treatment.

\section{Discussion}

IgG4-RD is a systemic disorder characterized by tendency to form tumefactive lesions, elevated levels of serum IgG4 (often but not always) and tissue infiltration by dense lymphocytes and IgG4-positive plasma cells in the affected organs. Autoimmune pancreatitis (AIP) is one of the most common disease features. Terumi Kamisawa and his colleague firstly proposed that AIP was not simply pancreatitis but that it was a pancreatic lesion involved in IgG4-RD with extensive organ involvement, and they defined IgG4-RD as a new clinicopathological entity in $2003{ }^{[7]}$. Actually, IgG4-RD has been described in virtually every organ system including pancreas, biliary duct, lacrimal/salivary glands, lymph node, lung, liver, kidney, thyroid gland, gastrointestinal tract, prostate, hypophysis, stomach, skin, aorta, joint, retroperitoneum, meninges and pleura ${ }^{[2-6]}$.

It is widely believed that hereditary susceptibility, autoimmunity and infectious agents are potential factors to lead to IgG4-RD ${ }^{[5]}$. Shigeyuki Kawa and his colleagues show that the frequencies of DRB $1 * 0405$ and DQB $1 * 0401$ are significantly higher in patients with AIP compared with chronic calcifying pancreatitis ${ }^{[8]}$. Their group also reveals that the HLA-linked genetic basis for AIP seems to be controlled by the DRB1*0405-DQB1*0401 mini-haplotype and the ABCF1 gene area ${ }^{[9]}$. Besides, plasminogen-binding protein (PBP) of Helicobacter pylori and ubiquitin-protein ligase E3 component nrecognin 2 (UBR2) expressed by acinar cells of the pancreas are homologous in peptide. $94 \%$ patients with autoimmune pancreatitis (33/35) can be detected antibodies against the PBP peptide, while can be detected by $5 \%$ patients with pancreatic cancer $(5 / 110)$, indicating that infectious factors and molecular mimicry may initiate IgG4-RD ${ }^{[10]}$. Moreover, the innate and the adaptive immune system might synergize to promote the progression of IgG4-RD and related tissue or organ fibrosis ${ }^{[11]}$. The microenvironment disorders are of critical importance for the pathogenesis of IgG4-RD, including immune cells including regulatory $T$ cell, regulatory $B$ cell, $T$ follicular helper 2 cell and M2 macrophage ${ }^{[12-14]}$. Inflammatory cytokines also participate in the IgG4-RD such as IFN- $\gamma$, IL-4, IL-10, IL-5, IL-13 and TGF- $\beta^{[11]}$. In fact, there is no specific pathogenic factors for IgG4-RD and its cognitive process is dynamic. Although genetic factor, infection and autoimmunity disorder may be involved in the pathogenesis of IgG4-RD, every hypothesis alone is not sufficient to explain it.

Since the incidence of IgG4-RD is quite low with estimation of 1.4 cases per 100,000 people (statistics from Japan) ${ }^{[15]}$, and sometimes diagnosis may be missed or erroneous. The multi-disciplinary involved 
method may be valuable for the diagnosis of IgG4-RD. There are no specific clinical symptoms or signs for IgG4-RD, usually common in other diseases or exerting symptoms of complications. Abdominal pain, nausea, vomiting and jaundice are more common in males, whereas swelling of the lacrimal glands and submandibular glands occurs more often in females. In males, the most commonly involved organs are salivary glands, pancreas and lacrimal glands, while they are salivary glands, lacrimal glands and sinuses in female ${ }^{[16]}$. The serum IgG4 level is elevated in most patients with increased ESR, CRP, serum IgG level and total IgE level, but there are still some patients presenting normal serum IgG4 levels (largely varied in different researches, from $2.5-40 \%{ }^{[17,18]}$. However, increased IgG4 level may occur in other diseases such as allergy and lymphoma. Organ enlargements of involved tissues are the most common imaging findings. If lungs are involved, honey-combing or ground-glass opacification, pulmonary nodules, bronchovascular bundle thickening may be found ${ }^{[17]}$. Both clinical and imaging manifestations are atypical, to some extent, which are likely to be misleading and confusing, especially for single or infrequent involved organ. Pathological examination is necessary and indispensible for the diagnosis of IgG4-RD. The typical microscopic findings are diffused lymphoplasmacytic infiltration with irregular and storiform fibrosis, and obliterative phlebitis, the former two of which occurs in most cases. Phlebitis without obliteration of the lumen and elevated numbers of eosinophils also exist sometimes, but no sensitive or specific effects for the diagnosis of IgG4-RD. Necrosis, discrete granuloma and extensive neutrophilic infiltration are the important clues to exclude IgG4-RD. Immunohistochemically, staining for IgG and IgG4 is very important. The absolute number of IgG4 positive plasma cells should reach a certain standard in corresponding organs or tissues, moreover, IgG4/lgG positive plasma cell ratio should be more than $40 \%{ }^{[18]}$. Although pathological examination is quite important for the diagnosis of IgG4-RD, it can't be confirmed by microscopy alone; multidisciplinary collaboration and comprehensive consideration with clinical and imaging manifestations along with laboratory tests is really important especially for single or infrequent involved organ.

To our knowledge, IgG4-RD rarely involves stomach. In the few reports available, almost every case is presenting multiple organs involvement accompanied by stomach related, and no case with fungal infection. We summarize all literature on the gastric involvement of IgG4-RD (Supplementary Table 1)]. In the above table, the majority of cases are multiple organs involved or confirmed by surgical specimen; no patient associates fungal infection. Microscopically, there are four zones in the base of the ulcer of surgical specimen: inflammatory exudates, fibrinoid necrosis, granulation tissue and mature fibrous tissue. However, pathologists always accept biopsy tissues surrounding the base of the ulcer in the clinical practice for fear that gastric perforation and unnecessary operation, which undoubtedly brings benefit for patients. At the same time, biopsy specimen make diagnosis for IgG4-RD involving stomach difficult, especially presenting with ulcer lesion. Pathologists must build up general impression based on the local findings. In this case, fungal infection makes diagnosis more confusing and challenging. Gastric mucormycosis may cause secondary changes for ulcer under microscope; besides, the identification of specific fungus will influence clinical decision and treatment methods.

\section{Conclusion}


As far as we know, it is the first report about IgG4-related disease manifesting as an ulcerated gastric cancer complicated with gastric mucormycosis. Multidisciplinary team discussion from gastroenterology, radiology and pathology department is necessary and indispensable for the disease diagnosis.

\section{Abbreviations}

IgG4-RD: IgG4-related disease; CT: computer tomography; PET-CT: positron emission tomographycomputed tomography; EGD: esophagogastroduodenoscopy; PPI: proton pump inhibitors; IHC: immunohistochemistry; ESR: erythrocyte sedimentation rate; ENA: extractable nuclear antigen; ANA: antinuclear antibody; ANCA: anti-neutrophil cytoplasmic antibodies; PR3: anti-protease 3; MPO: antimyeloperoxidase; GBM: glomerular basement membrane antibody; PCR: polymerase chain reaction; AIP: autoimmune pancreatitis; PBP: plasminogen-binding protein; UBR2: ubiquitin-protein ligase E3 component n-recognin 2.

\section{Declarations}

\section{Acknowledgements}

We thank Mrs Meirong Li of The Second Xiangya Hospital, Central South University for performing the IHC analyses.

\section{Authors' contributions}

Songqing Fan designed and revised the manuscript. Yuting Zhan wrote the manuscript and made the tables. Yang Yang helped to handle the surgical specimen and collect pathological figures. Hongmei Zheng and Yuting Zhang helped to collect clinical statistics. Li Gu conducted esophagogastroduodenoscopy and provided pictures. Dongliang Chen provided the slides of local hospital and participated in the diagnosis of this case.

\section{Funding}

The work was supported by the National Natural Sciences Foundations of China (No: 81472773 and 81773218)

\section{Availability of data and materials}

The dataset supporting the conclusions of this article is included within the article(and its additional file).

\section{Consent for publication}

The patients were consent with this study.

\section{Competing interests}


The authors have no conflict of interest to disclose.

\section{Author details}

1: Department of Pathology, The Second Xiangya Hospital, Central South University, Changsha, Hunan, 410011, China

2: Department of Digestive Medicine, The Second Xiangya Hospital, Central South University, Changsha, Hunan, 410011, China

3: Department of Pathology, the Affiliated Zhuzhou Hospital of XiangYa Medical college of Central South University, Zhuzhou, Hunan, 412007, China

\section{References}

[1] Kamisawa T, Zen Y, Pillai S, Stone JH. IgG4-related disease. Lancet. 2015. 385(9976): 1460-1471.

[2] Avincsal MO, Zen Y. The Histopathology of IgG4-Related Disease. Curr Top Microbiol Immunol. 2017. 401: 45-60.

[3] Okazaki K, Umehara H. Current Concept of IgG4-Related Disease. Curr Top Microbiol Immunol. 2017. 401: 1-17.

[4] Cheong HR, Lee BE, Song GA, Kim GH, An SG, Lim W. Immunoglobulin G4-Related Inflammatory Pseudotumor Presenting as a Solitary Mass in the Stomach. Clin Endosc. 2016. 49(2): 197-201.

[5] Vaglio A, Zwerina J. IgG4-related disease. N Engl J Med. 2012. 366(17): 1646; author reply 1646-7.

[6] Leporati P, Fonte R, Chiovato L. IgG4-related disease. N Engl J Med. 2012 Apr 26;366(17):1645; author reply 1646-1647.

[7] Kamisawa T, Funata N, Hayashi Y, et al. A new clinicopathological entity of IgG4-related autoimmune disease. J Gastroenterol. 2003. 38(10): 982-984.

[8] Kawa S, Ota M, Yoshizawa K, et al. HLA DRB10405-DQB10401 haplotype is associated with autoimmune pancreatitis in the Japanese population. Gastroenterology. 2002. 122(5): 1264-1269.

[9] Ota $\mathrm{M}$, Katsuyama $\mathrm{Y}$, Hamano $\mathrm{H}$, et al. Two critical genes (HLA-DRB1 and ABCF1)in the HLA region are associated with the susceptibility to autoimmune pancreatitis. Immunogenetics. 2007. 59(1): 45-52.

[10] Frulloni L, Lunardi C, Simone R, et al. Identification of a novel antibody associated with autoimmune pancreatitis. N Engl J Med. 2009. 361(22): 2135-42.

[11] Della-Torre E, Lanzillotta M, Doglioni C. Immunology of IgG4-related disease. Clin Exp Immunol. 2015. 181(2): 191-206. 
[12] Uchida K, Okazaki K. Roles of Regulatory T and B Cells in IgG4-Related Disease. Curr Top Microbiol Immunol. 2017. 401: 93-114.

[13] Akiyama M, Suzuki K, Yasuoka H, Kaneko Y, Yamaoka K, Takeuchi T. Follicular helper T cells in the pathogenesis of IgG4-related disease. Rheumatology (Oxford). 2018. 57(2): 236-245.

[14] Kawakami T, Mizushima I, Yamada K, et al. Abundant a proliferation-inducing ligand (APRIL)producing macrophages contribute to plasma cell accumulation in immunoglobulin G4-related disease. Nephrol Dial Transplant. 2018.

[15] Kanno A, Masamune A, Okazaki K, et al. Nationwide epidemiological survey of autoimmune pancreatitis in Japan in 2011. Pancreas. 2015. 44(4): 535-539.

[16] Wang L, Zhang P, Zhang X, et al. Sex disparities in clinical characteristics and prognosis of immunoglobulin G4-related disease: a prospective study of 403 patients. Rheumatology (Oxford). 2018 .

[17] Lin W, Lu S, Chen H, et al. Clinical characteristics of immunoglobulin G4-related disease: a prospective study of 118 Chinese patients. Rheumatology (Oxford). 2015. 54(11): 1982-1990.

[18] Deshpande V, Zen Y, Chan JK, et al. Consensus statement on the pathology of IgG4-related disease. Mod Pathol. 2012. 25(9): 1181-1192.

[19] Seo HS, Jung YJ, Park CH, Song KY, Jung ES. IgG4-related Disease in the Stomach which Was Confused with Gastrointestinal Stromal Tumor (GIST): Two Case Reports and Review of the Literature. J Gastric Cancer. 2018. 18(1): 99-107.

[20] Bulanov D, Arabadzhieva E, Bonev S, et al. A rare case of IgG4-related disease: a gastric mass, associated with regional lymphadenopathy. BMC Surg. 2016. 16(1): 37.

[21] Na KY, Sung JY, Jang JY, et al. Gastric nodular lesion caused by IgG4-related disease. Pathol Int. 2012. 62(10): 716-718.

[22] Inoue D, Yoneda N, Yoshida K, et al. Imaging and pathological features of gastric lesion of immunoglobulin G4-related disease: A case report and review of the recent literature. Mod Rheumatol. 2019. 29(2): 377-382.

[23] Inoue K, Okubo T, Kato T, et al. IgG4-related stomach muscle lesion with a renal pseudotumor and multiple renal rim-like lesions: A rare manifestation of IgG4-related disease. Mod Rheumatol. 2018. 28(1): 188-192.

[24] Bateman AC, Sommerlad M, Underwood TJ. Chronic gastric ulceration: a novel manifestation of IgG4-related disease. J Clin Pathol. 2012. 65(6): 569-570. 
[25] Frydman J, Grunner S, Kluger Y. IgG4-related disease manifesting as an acute gastric-pericardial fistula. World J Gastroenterol. 2014. 20(44): 16782-16785.

[26] Li M, Zhou Q, Yang K, et al. Rare case of Helicobacter pylori-positive multiorgan IgG4-related disease and gastric cancer. World J Gastroenterol. 2015. 21(11): 3429-3434.

[27] Fujita T, Ando T, Sakakibara M, Hosoda W, Goto H. Refractory gastric ulcer with abundant IgG4positive plasma cell infiltration: a case report. World J Gastroenterol. 2010. 16(17): 2183-2186.

[28] Yang L, Jin P, Sheng JQ. Immunoglobulin G4-related disease (IgG4-RD) affecting the esophagus, stomach, and liver. Endoscopy. 2015. 47 Suppl 1 UCTN: E96-7.

[29] Mine S, Ozawa E, Ohnita K, Abe K, Nakao K. A case of IgG4-related disease presenting with multiple gastric submucosal tumors. Gastrointest Endosc. 2018. 87(2): 608-610.

[30] Kaji R, Okabe Y, Ishida Y, et al. Autoimmune pancreatitis presenting with IgG4-positive multiple gastric polyps. Gastrointest Endosc. 2010. 71(2): 420-422.

\section{Figures}
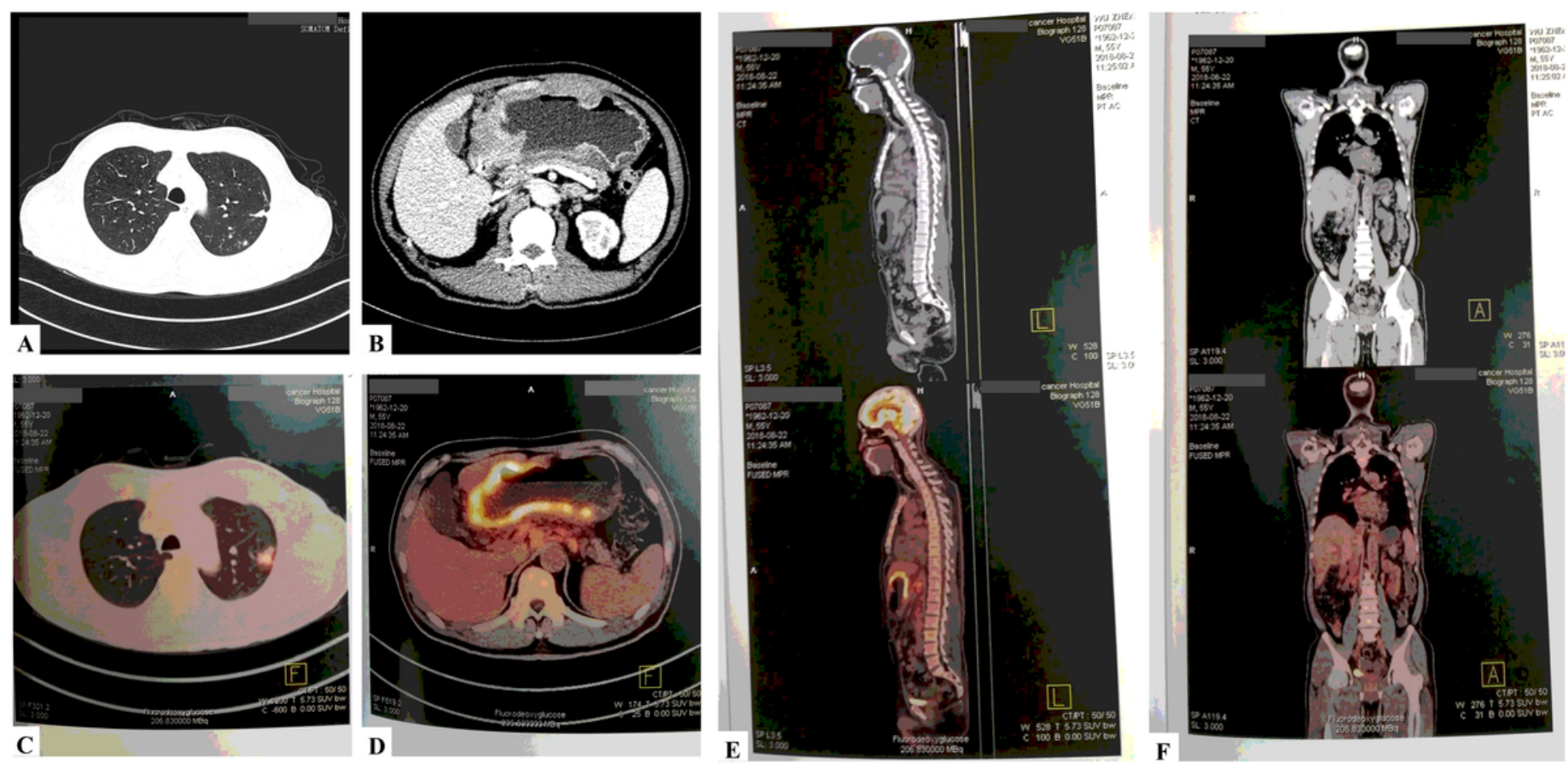

Zhan Y, et al, Figure 1

Figure 1

Imaging manifestations of this rare case. Contrast-enhanced CT indicated that gastric wall thickening, perigastric and retroperitoneal lymph node enlargement, and nodule in left upper lung with double lungs 
exudation (A, B); PET-CT showed radioactive accumulations in the above position as well as retroperitoneal lymph nodes (C-F).
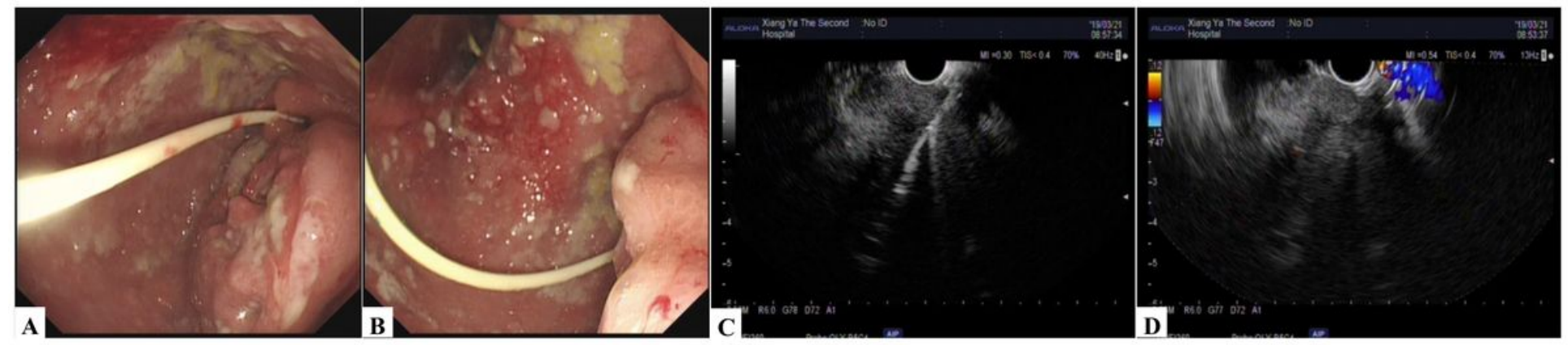

Zhan Y, et al, Figure 2

Figure 2

Esophagogastroduodenoscopy images of this rare case. A huge ulcer was found by esophagogastroduodenoscopy (A, B); Ultrasound gastroscope showed gastric wall thickening of antrum $(\mathrm{C}, \mathrm{D})$.

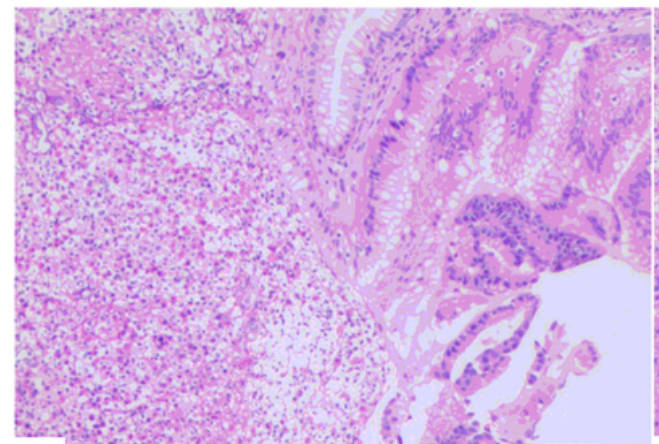

A $\quad 50$ B 4.

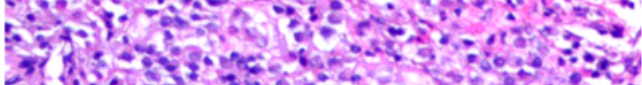

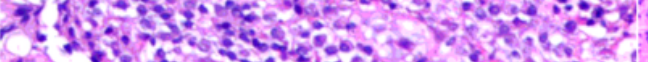

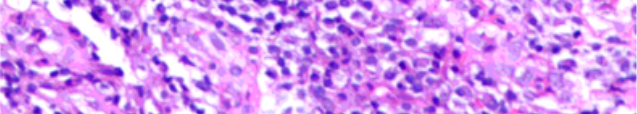

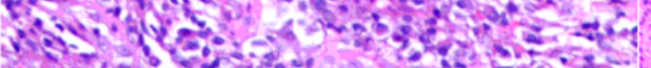

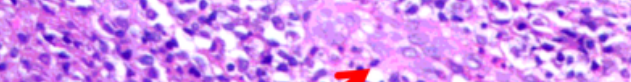

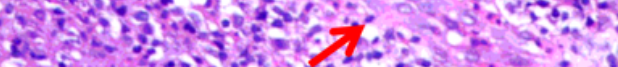
$35,4506=2$

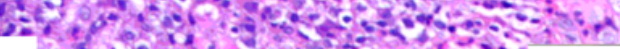

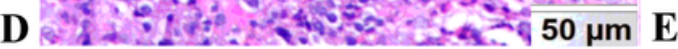

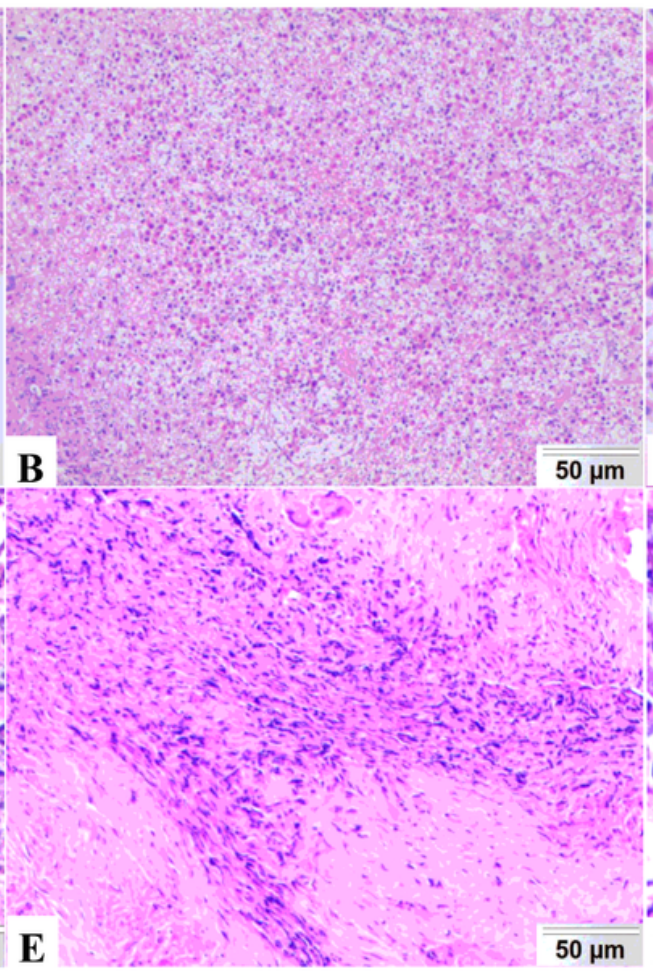

$50 \mu \mathrm{m}$
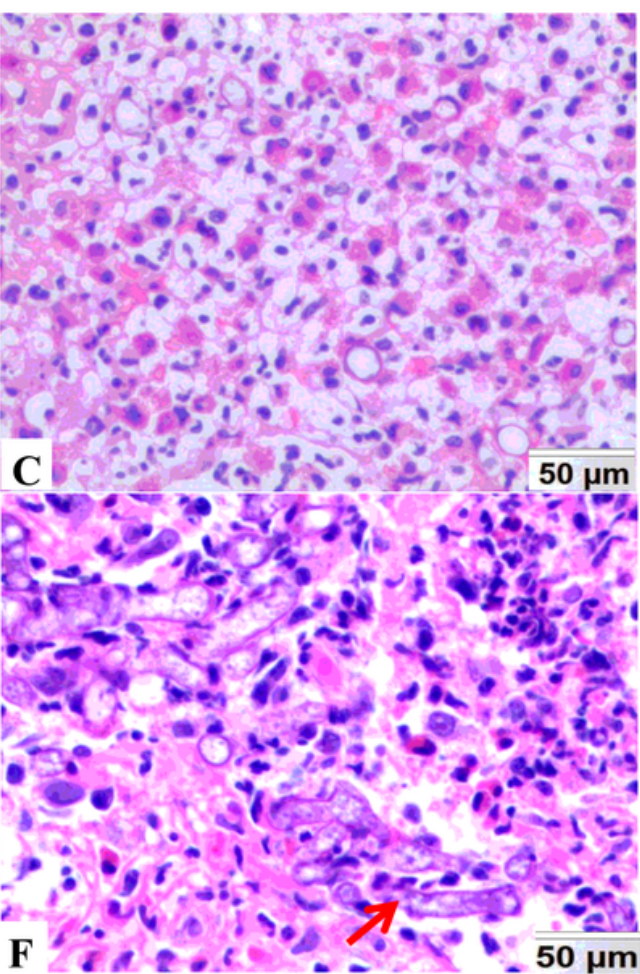

Zhan Y, et al, Figure 3

\section{Figure 3}

The HE staining for the typical microscopic findings of this case (biopsy specimen). Microscopically, ulcer $(A, 100 x)$ and inflammatory granulation $(B, 100 x)$ could be seen with plasma cells in sheets and lymphocytes $(C, 200 x)$, inflammation of vascular wall (D, 200x), formation of new fibrous tissue (E, 100x), and hyphae and spores also be observed in inflammatory necrosis ( $F, 400 x)$. 

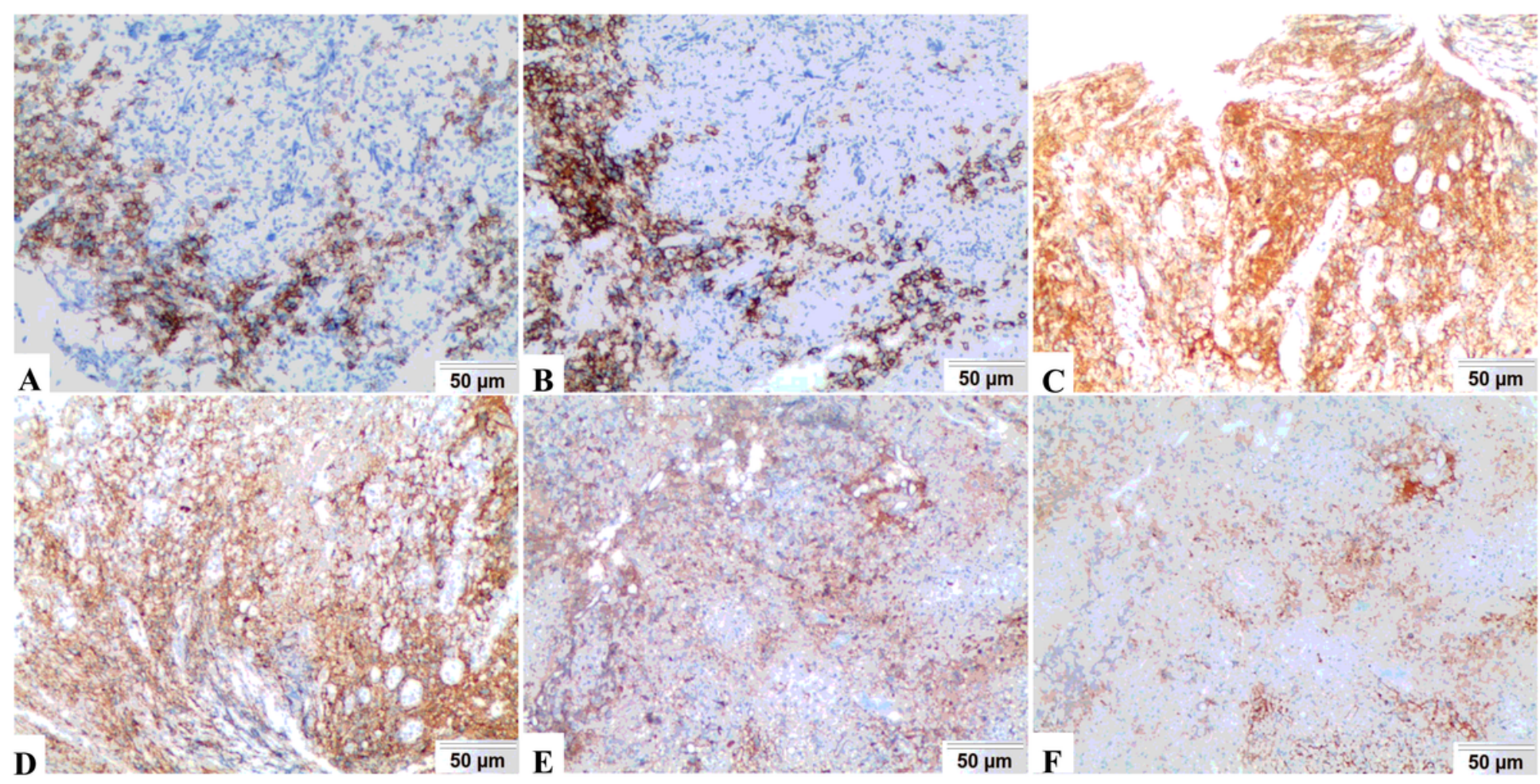

Zhan Y, et al, Figure 4

Figure 4

The typical IHC staining for this case (biopsy specimen). The plasma cells had immunohistochemically strong staining for CD38 (A, 100x), CD138 (B, 100x), kappa (C, 100x), lambda (D, 100x), lgG (E, 100x), and $\lg \mathrm{G} 4(\mathrm{~F}, 100 \mathrm{x})$.

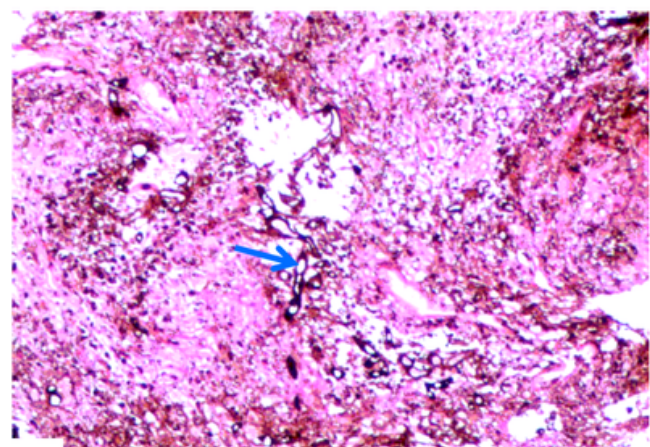

A the s.

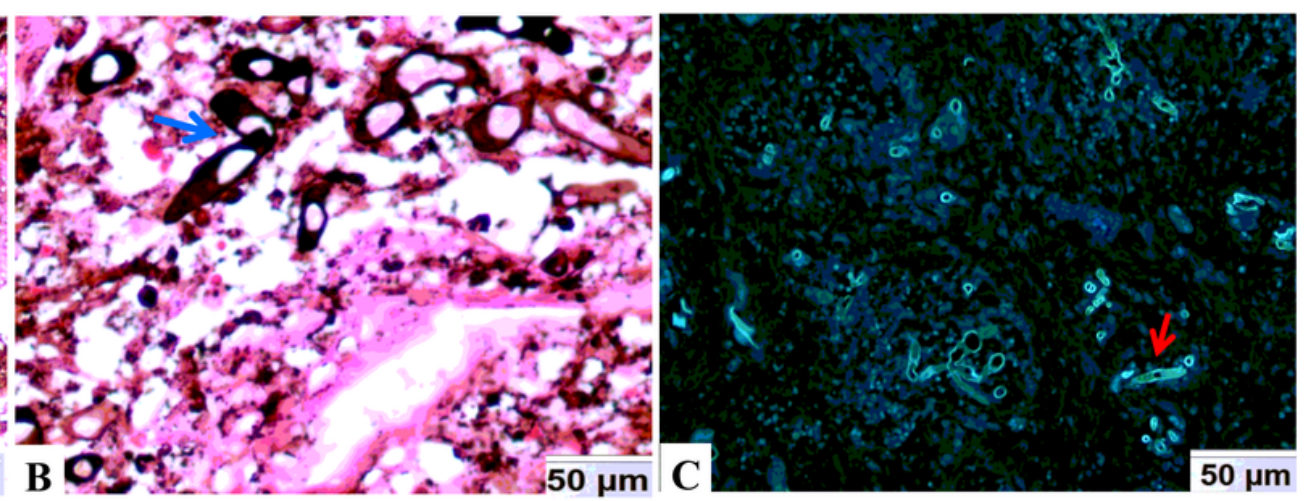

Zhan Y, et al, Figure 5

\section{Figure 5}

The typical images for hyphae and spores of mucormycosis of this case. Gomori's methenamine silver staining for hyphae and spores of mucormycosis (A, 100x, B, 400x). Immunofluorescence and confocal laser-scanning microscopy were used to characterize hyphae and spores of mucormycosis (C, 200x).

\section{Supplementary Files}


This is a list of supplementary files associated with this preprint. Click to download.

- CAREchecklist81620212.docx

- Figure1S.tif

- SupplementaryTable1.docx 